\title{
Detection OF CONCEALED WEAPONS IN X-RAY IMAGES USING FUZZY K-NN
}

\author{
Dr. Mohamed Mansoor Roomi ${ }^{\# 1}$ R.Rajashankari ${ }^{\# 2}$ \\ Department of Electronics and Communication Engineering, Thiagarajar College of \\ Engineering,Madurai \\ 1 smmroomi atce.edu \\ ${ }^{2}$ r.rajasankari@gmail.com
}

\begin{abstract}
Scanning baggage by $x$-ray and analysing such images have become important technique for detecting illicit materials in the baggage at Airports. In order to provide adequate security, a reliable and fast screening technique is needed for baggage examination.This paper aims at providing an automatic method for detecting concealed weapons, typically a gun in the baggage by employing image segmentation method to extract the objects of interest from the image followed by applying feature extraction methods namely Shape context descriptor and Zernike moments. Finally the objects are classified using fuzzy KNN as illicit or non-illicit object.
\end{abstract}

\section{KEYWORDS}

Aviation security, Shape Context Descriptor, Zernike Moments, Nearest Neighbour Classifier

\section{INTRODUCTION}

$\mathrm{X}$-ray imaging is an important technology in many fields from inspection of delicate objects to weapon detection at security checkpoints [1].To achieve higher threat detection rates during inspection of X-ray luggage scans is a pressing and sought after goal for airport security personnel. The Baggage inspection system used in airport ensures security of the passengers. The process of identifying the contents of each bag and the methods adopted by terrorists for hiding the threat objects are complicated, the existing luggage inspection system do not reveal $100 \%$ of threat items. Further an object inside a bag may be in any position, it may be rotated so an algorithm whist is rotational, translational invariant should be used for providing accurate results. In addition, the threat item is superimposed by other objects in the bag, the harder it becomes to detect it (effect of superposition). The passenger's baggage may contain threat items such as handgun, bomb, grenade,etc which must be detected efficiently so the human operators must be assisted by an weapon detection system. Advanced security screening systems are becoming increasingly used to aid airport screeners in detecting potential threat items [2]. Unfortunately, most airport screening is still based on the manual detection of potential threat objects by human experts. In response to this, security training is relying heavily on the object recognition test (ORT) as a means of qualifying human airport luggage screeners [4].In order to provide appropriate security, a much more sophisticated, reliable, and fast screening technique is needed for passenger identification and baggage examination. Automatic threat detection is an important application in x-ray scene analysis. Understanding x-ray images is a challenging task in computer 
vision and an automatic system should be developed that consumes less time for processing and performs accurately with reduced false positive results.

Although several X-ray technology based automatic systems exist for threats detection, only a few of these systems make use of the well established pattern recognition and machine learning techniques.On the other hand, several approaches based on Classifier have

been proposed to detect weapons[3].Additionally, the importance of image enhancement and pseudo-coloring[5] to help aid decision making by human is now a recognized area of critical need. Also, the system should provide automatic detection of potential threat objects.

\section{RELATED WORK}

For the detection of threat items, many types of imaging system exist. X-ray imaging systems and MMW (Millimetre wave imaging) are used and x-ray imaging system is widely used for carry-on bags. The techniques used for analysing these x-ray images are pseudo-coloring and segmentation based techniques. Pseudo-coloring [1] process is the one in which the objects inside the bag are given different colors based on their material type. In segmentation based methods, the x-ray images are segmented to extract the objects of interest. Using these methods, satisfactory results are produced and assisted human for detecting the threat items. X-ray photons, however, penetrate most materials. As a result, all objects along an $\mathrm{x}$-ray path attenuate the $\mathrm{x}$-ray and contribute to the final measured intensity. In the x-ray community, a common way of disambiguating objects is through CT reconstruction [7]. This is typically obtained through the filtered back-projection algorithm. Although several X-ray technology based automatic systems exist for threats detection [8], only a few of these systems make use of the well established pattern recognition and machine learning techniques [9, 10, 11, and 12]. New X-ray imaging systems at airports use dual-energy analysis to estimate the atomic numbers of materials in the passenger baggage. This method obtains a measure of the density and thickness of the material.

\section{PROPOSED WORK.}

The image is converted to binary image by choosing the threshold as the mean of the two peaks of bimodal histogram and the objects are labelled. The area of each object is computed and the values are sorted. The mean value is calculated and is set as threshold to collect the objects of interest. Object boundary is extracted and shape feature extraction algorithm is implemented. The classifier is trained with the extracted features and the object is classified as object or non-object.
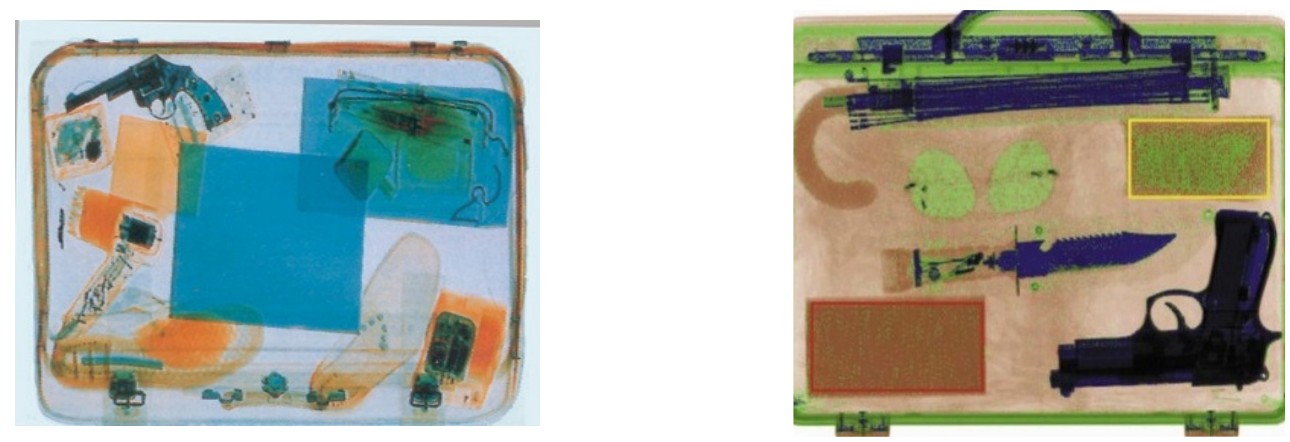

Figure 1 SampleX-ray Images 
Figure 1 shows some of the x-ray images considered in this work.Figure 2 shows the overall frame work for the approach.

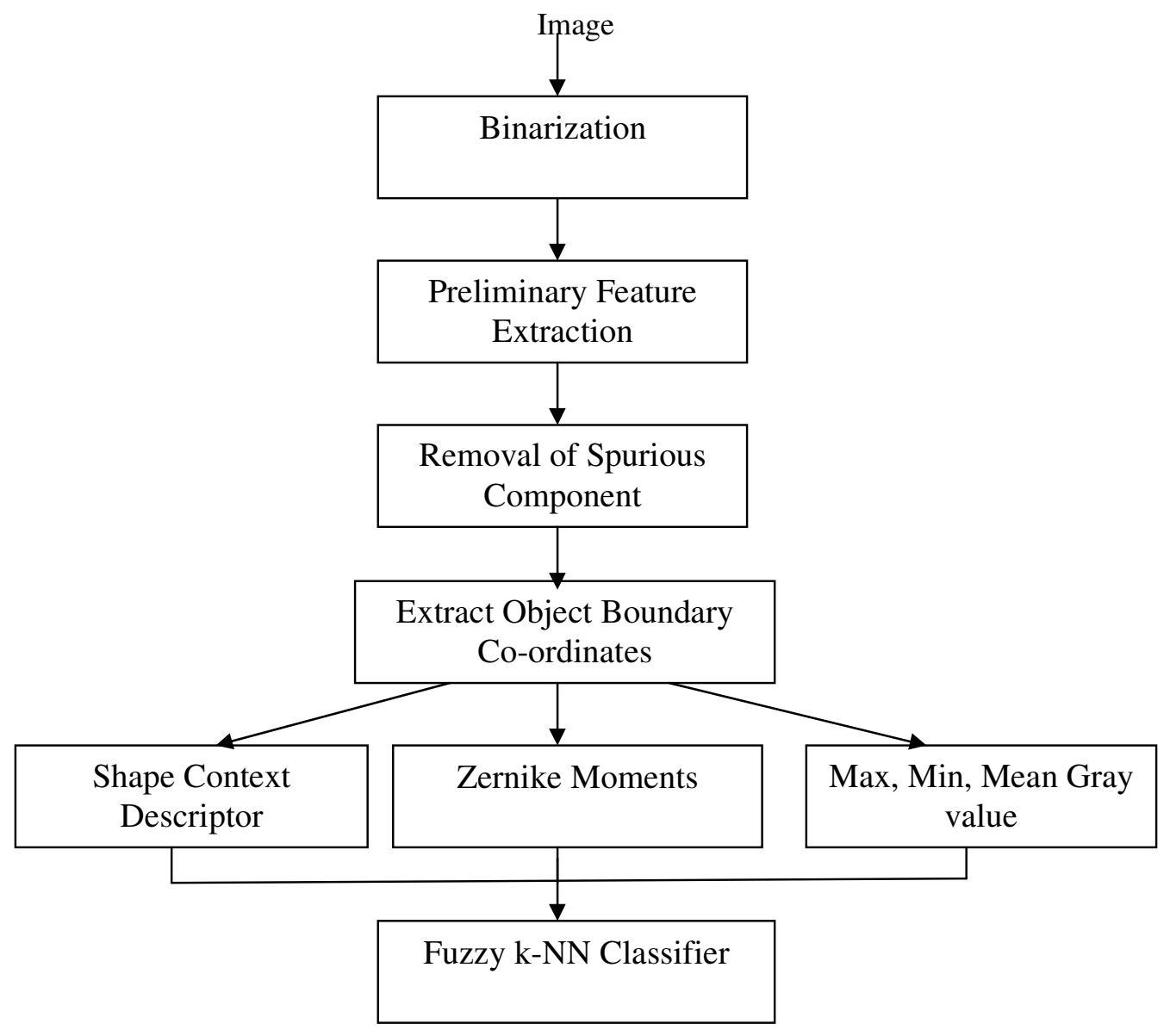

Figure 1 Flow chart of proposed work

\subsection{FEATURE EXTRACTION}

\subsubsection{SHAPE CONTEXT DESCRIPTOR}

Shape is not the only, but a very powerful descriptor of image content .Shape is almost certainly the most important property that is perceived about objects. Shape provides more information about the object than other features and can be used in object recognition. Using shape as a attribute provide more accurate and reliable results .Addressing objects based on their shape is unique. Shape is an important cue as it captures a prominent element of an object.. Ideally, a good shape descriptor has the following desirable properties1)Discrimination should be high;2)Efficient matching;3)Compact representation;4)Efficient Feature Extraction;5)Invariance to shape representation;6)Invariance to similarity transformation;7)Invariance to shape degeneracies and noises.

Shape context is a shape descriptor proposed by Serge Belongie and Jitendra Malik[13]. The shape context is anticipated to be a way of describing shapes that allows for measuring shape similarity and the recovering of point correspondences.It characterize a particular 
point location on the shape.The fundamental idea is to pick $n$ points on the contours of a shape. For each point $p_{i}$ on the shape, consider the $n-1$ vectors obtained by connecting $p_{i}$ to all other points. The set of all these vectors is a rich description of the shape localized at that point but is far too detailed. The key initiative is that the distribution over relative positions is a robust, compressed, and very discriminative descriptor. So, for the point $p_{i}$, the coarse histogram of the relative coordinates of the remaining $n-1$ points, Concretely, for a point $\mathrm{p}_{\mathrm{i}}$ on the shape, compute a coarse histogram hi of the relative coordinates of the remaining $n-1$ points

$$
h_{i}^{k}=\#\left\{q \neq p_{i}:\left(q-p_{i}\right) \in \operatorname{bin}(k)\right\}
$$

is define to be the shape context of $p_{i}$. The bins are normally taken to be uniform in logpolar space. In the absence of background clutter, the shape context of a point on a shape can be made invariant under uniform scaling of the shape as a whole. This is accomplished by normalizing all radial distances by the mean distance $\alpha$ between the $\mathrm{n}^{2}$ point pairs in the shape. Consider the shape of the alphabet fig.3 (a).sampled edge points of the shape. A log polar histogram bin as shown in fig. 3 (b) is overlaid on any sampled boundary point. Belongie et al. have used 12 bins for $\log r$ and 5 bins for angle $\theta$. As illustrated in Fig. 1, shape contexts is computed for each point in the shape and will be unique for each point and similar shapes will have similar shape context.Translational invariance come naturally to shape context. Scale invariance is obtained by normalizing all radial distances by the mean distance between all the point pairs in the shape.

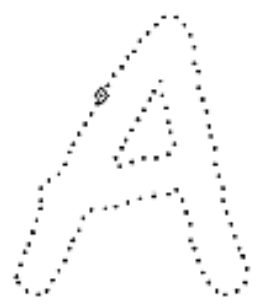

(a)

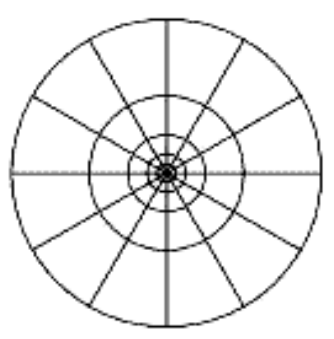

(b)

Figure 2 Shape Context Computation

\subsubsection{ZERNIKE MOMENTS}

Moments have been widely used in image processing applications through the years. For both contour and region of a shape, one can use moment's theory to analyse the object. Geometrical, central and normalized moments were for many decades the only family of applied moments. The main disadvantage of these descriptors was their disability to fully describe an object in a way that, using the moments set, the reconstruction of the object could be possible. In other words they are not orthogonal. Zernike comes to fill this gap, by introducing a set of complex polynomials, which form a complete orthogonal set over the interior of the unit circle, $x^{2}+y^{2}=1$.

These polynomials have the form

$$
V_{n m}(x, y)=V_{n m}(\rho, \theta)=R_{n m}(\rho) \exp (j m \theta)
$$


Where $n$ is a non-negative integer $m$ is a non zero integer subject to the constraints $n$-|m| even and $|m| \leq n, \rho$ is the length of vector from the origin $(\bar{x}, \bar{y})$ to the $\operatorname{pixel}(x, y), \theta$ the angle between vector $\rho$ and $x$ axis in counter-clockwise direction. $R_{n m}(\rho)$ is the Zernike radial polynomials in $(\rho, \theta)$ polar coordinates

$R_{n,-m}(\rho)=R_{n m}(\rho)$

The Zernike moment of order $n$ with repetition $m$ for a continuous image function $\mathrm{f}(x, y)$, that vanishes outside the unit disk is

$$
Z_{n m}(x, y)=\frac{n+1}{\pi} \iint_{x^{2}+y^{2} \leq 1} f(x, y) V_{n m}^{*}(\rho, \theta) d x d y
$$

For a digital image, the integrals are replaced by summations to get

$$
Z_{n m}=\frac{n+1}{\pi} \sum_{x} \sum_{y} f(x, y) V_{n m}^{*}(\rho, \theta), x^{2}+y^{2} \leq 1
$$

Suppose that one knows all moments $Z_{n m}$ of $f(x, y)$ up to a given order $n_{\max }$. It is desired to reconstruct a discrete function $\hat{f}(x, y)$ whose moments exactly match those of $\mathrm{f}(\mathrm{x}, \mathrm{y})$ up to the given order $n_{\max }$. Zernike moments are the coefficients of the image expansion into orthogonal Zernike polynomials. By orthogonality of the Zernike basis

$\hat{f}(x, y)=\sum_{n=0}^{n_{\max }} \sum_{m} Z_{n m} V_{n m}(\rho, \theta)$

Since Zernike moments are only rotationally invariant, additional properties of translation and scale invariance should be given to these moments in some way. We can introduce translation invariance in the Zernike moments by converting the absolute pixel coordinates as follows

$$
\left(\begin{array}{l}
x \\
y
\end{array}\right) \rightarrow\left(\begin{array}{c}
x-X_{0} \\
y-Y_{0}
\end{array}\right)
$$

$$
\text { Where } \begin{aligned}
X_{0} & =\frac{m_{10}}{m_{00}} \\
Y_{0} & =\frac{m_{01}}{m_{00}}
\end{aligned}
$$

are the centroid coordinates of the object (with $m$ denoting the geometrical moment).

Scaling invariance can be achieved by normalizing the Zernike moments with respect to the geometrical moment $\mathrm{mOO}$ of the object. The resulting moments are derived from the following equation 


$$
Z_{n m}^{\prime}=\frac{Z_{n m}}{m_{00}}
$$

where $Z_{n m}$ are the Zernike moments computed by using equation (4.4).Since Zernike basis functions take the unit disk as their domain, this disk must be specified before moments can be calculated.

In the implementation, all the shapes are normalized into a unit circle of fixed radius. The unit disk is then centred on the shape centroid. This makes the obtained moments scale and translation invariant. Rotation invariance is achieved by only using magnitudes of the moments. Rotation invariance is achieved by only using magnitudes of the moments. The magnitudes are then normalized by dividing them by the mass of the shape.

The similarity between two shapes indexed with Zernike moments descriptors is measured by the Euclidean distance between the two Zernike moments vectors. The computation of ZMD does not need to know boundary information, making it suitable for more complex shape representation. Like Fourier descriptors, Zernike moments descriptors can be constructed to arbitrary order, this overcomes the drawback of geometric moments in which higher order moments are difficult to construct. However, Zernike moments descriptors lose the perceptual meanings as those reflected in Fourier descriptors and geometric moments. Besides, ZMD does not emphasize shape boundary features which are important features of a shape. Zernike moments have many advantages such as rotation invariance(the magnitudes of Zernike moments are invariant to rotation), robustness(they are robust to noise and minor variations in shape) and expressiveness(since the basis is orthogonal, they have minimum information redundancy).

\subsection{Classification}

Once significant features are extracted from X-ray images, a good classification technique is needed to identify the target object with a quantified confidence level so that this information can assist the security operator in making an appropriate response. Classifiers ranging from KNN to the Artificial Neural Networks (ANNs)are used for image classification, shape recognition, and image retrieval. However the classification rate in $\mathrm{x}$-ray luggage scanning is well below satisfactory levels.

\subsubsection{FUZZY K-NN CLASSIFIER}

Fuzzy k-nearest neighbor is a classification technique, which provides the simplicity and the practicability of classical K nearest neighbor and also the advantages of using fuzzy logic. Fuzzy sets are sets whose elements have degrees of membership. In classical set theory, the membership of elements in a set is assessed in binary terms according to a bivalent condition - an element either belongs or does not belong to the set. By contrast, fuzzy set theory permits the gradual assessment of the membership of elements in a set; this is described with the aid of a membership function. This algorithm assigns membership as a function of the object's distance from its Knearest neighbours and the memberships in the possible classes. The main algorithm is very similar to K-NN. In the training procedure, the sample objects are located to the feature vector space and these samples are initialized in a fuzzy state. In the classification phase, for each new object $\mathrm{x}$, it's $\mathrm{K}$ nearest neighbors are detected and then class membership values are calculated according to the following formula: 
$u_{i}(x)=\frac{\sum_{j=1}^{K} u_{i j} W_{j}}{\sum_{j=1}^{K} W_{j}}$

Where, $u_{i j}$ is the $i^{\text {th }}$ class membership value of neighbor ${ }_{j}$ and $W_{j}$ is the weight of the neighbor ${ }_{j}$ given by

$W_{j}=\frac{1}{(\text { dis } \tan c e)^{2 / m-1}}$

A distance measure $d\left(i_{1}, i_{2}\right)$ between any pair $a_{1}=\left(a_{1,1}\right.$

,$\left.a_{1, k}\right) ; a_{2}=\left(a_{2,1}\right.$

, $\left.\mathrm{a}_{2, \mathrm{k}}\right)$ of instances Euclidean distance is given by

$d_{2}\left(a_{1}, a_{2}\right)=\sqrt{\sum_{j=1}^{k}\left(a_{1, j}-a_{2, j}\right)^{2}}$

The value of $\mathrm{m}$, used to scale the effect of the distance between $x$ and neighbor ${ }_{j}$, is entirely arbitrary. As $m$ approaches to \pm infinity, the results of classifier approach to K-nn.

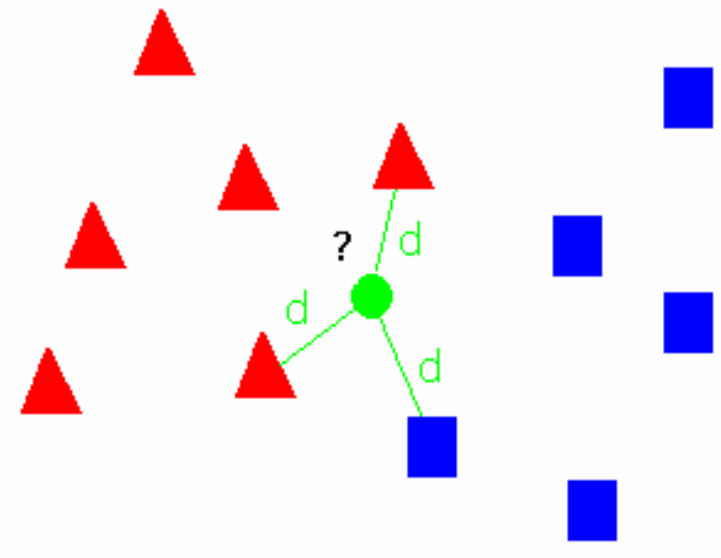

Figure 3 A Classification Example

Figure 3 shows A classification example of an unlabeled object with using fuzzy $\mathrm{k}$ nearest neighbor algorithm. In the figure (a), the unlabeled green circular object's 3 neighbors are at the same distance.

Fuzzy K-NN approach reduces the disadvantages of traditional K-NN approach and also it is a simple and effective solution for classification. 


\section{RESULTS AND DISCUSSION}

In this work the X-ray images of bags which are taken in the airport were considered. Size of the images used for processing is $310 \mathrm{X} \mathrm{1035}$. To obtain the optimum result in classification an efficient algorithm is used.In this project, feature extraction technique is applied to the input pre-processed image to extract features .A total of 15 images are taken among which seven images are with weapon and the remaining images are without weapon. The classifier which is used in this project classify efficiently. The classifier is initially trained for all the images. The training images with a new set of two images which is not included in the training is considered for testing. Figure 4 shows the objects of interest extracted after preliminary feature extraction process. A set of training and testing images considered for classification is shown in Figure 5 and Figure 6 respectively.

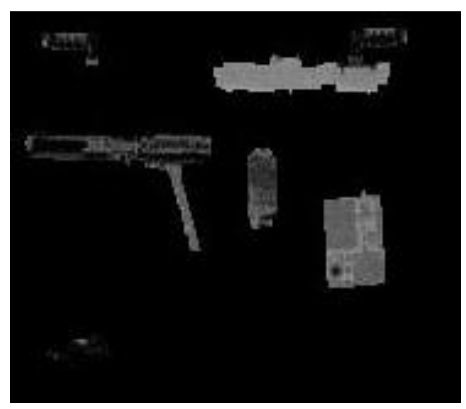

Figure 4 Objects Of Interest Extracted
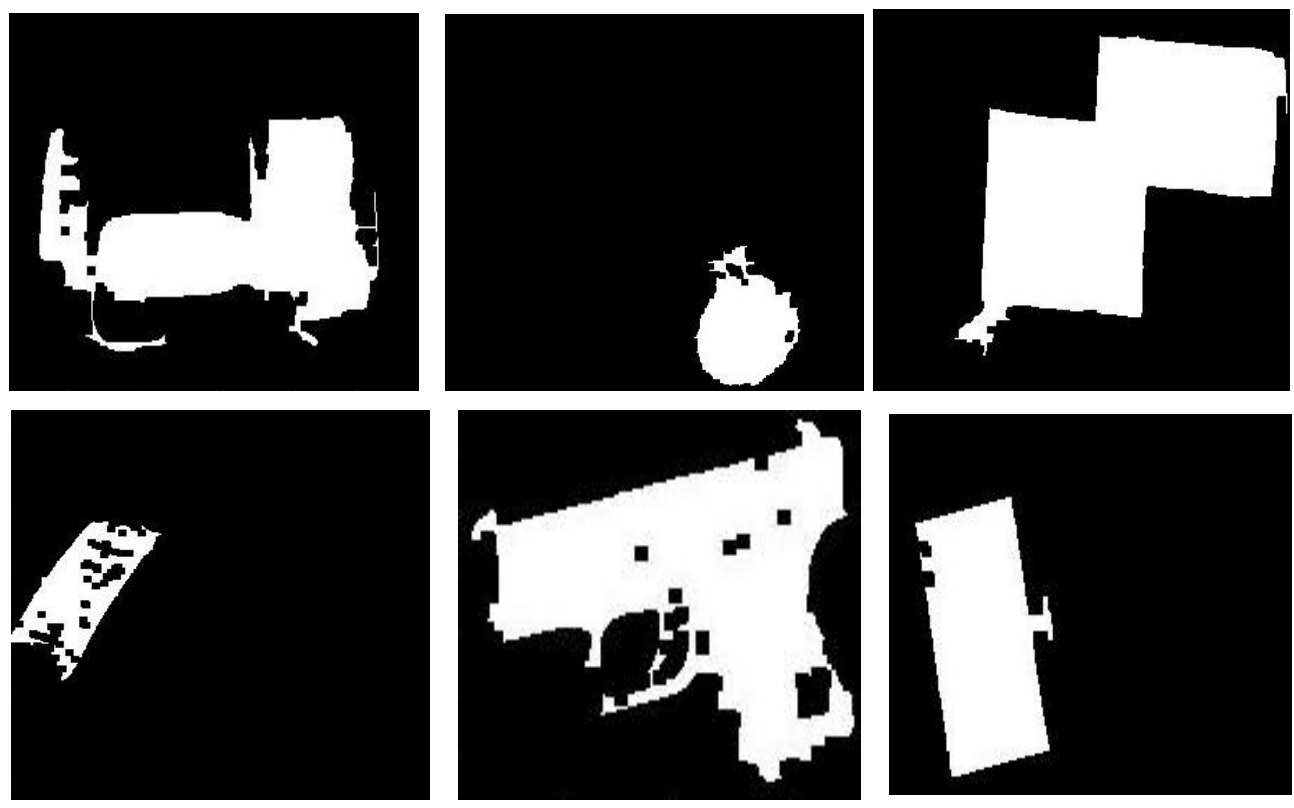

Figure 5 Training Images 

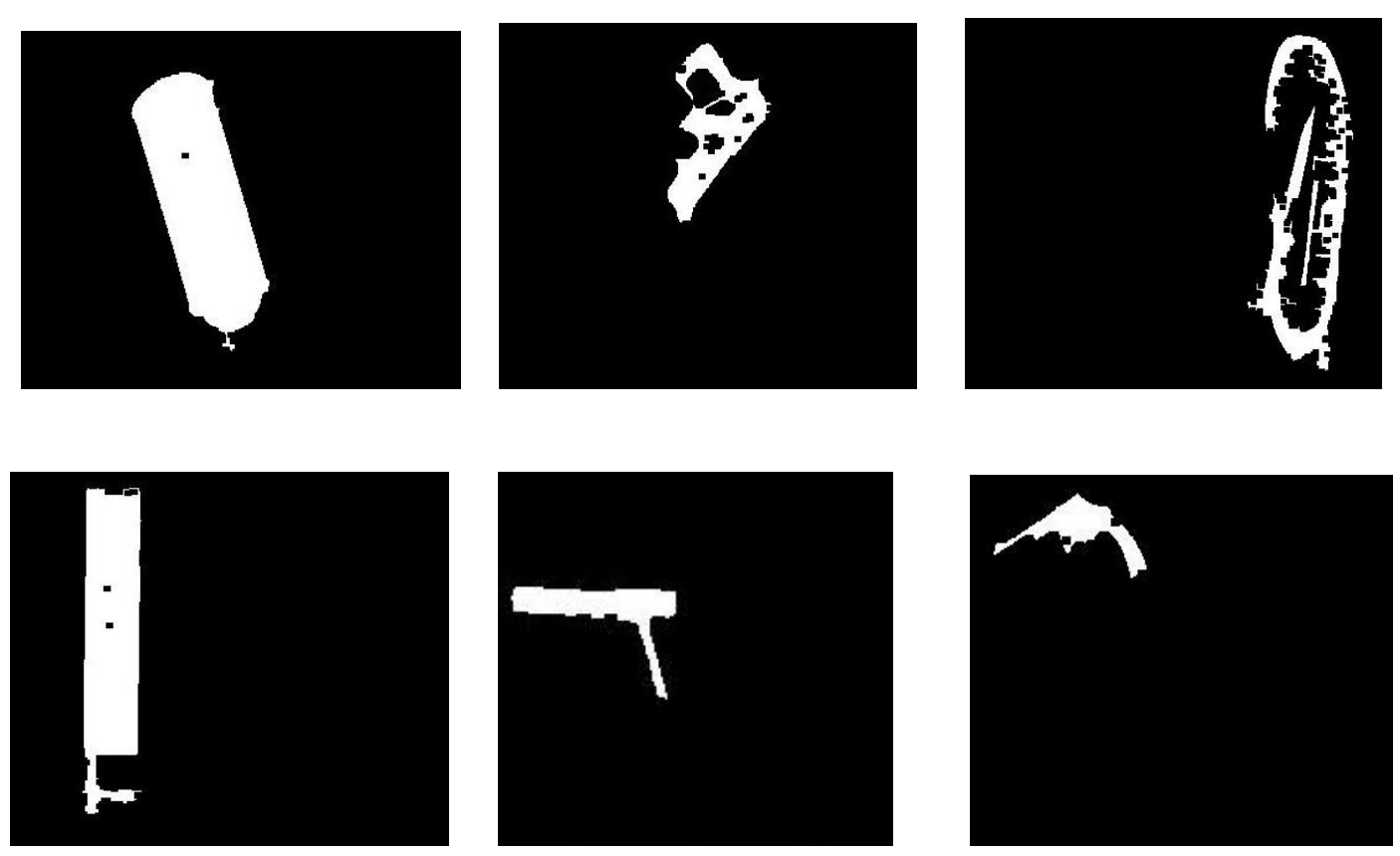

Figure 6 Testing Images

Figure 7 shows the detected weapon in the x-ray images considered.

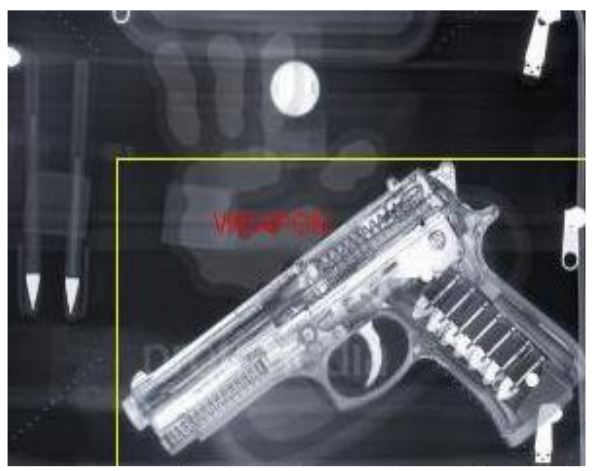

Figure 7 Detected Weapon

\section{CONCLUSION}

In this work, a fuzzy KNN based classifier has been presented to detect concealed weapon by capturing and analysing x-ray images. This proposed work relies on reliable features like Shape context descriptor and Zernike moments to detect concealed weapons. The proposed method performs satisfactorily and the future work involves classification of detected weapons. 
International Journal of Computer Science, Engineering and Information Technology (IJCSEIT), Vol.2, No.2, April 2012

\section{References}

[1] Abidi, B., Y. Zheng, A. Gribok, and M. Abidi,. Improving Weapon Detection in Single Energy XRay Images Through Pseudocoloring.

[2] N.E.L. Shanks and A.L.W. Bradley, Handbook of Checked Baggage Screening: Advanced Airport Security Operation, WileyBlackwell, 2004.

[3] Steve Green, Michael Blumenstein, Vallipuram Muthukkumarasamy and Jun Jo School of Information Technology, Investigation of a classification-based technique to detect Illicit objects for aviation security.

[4] D. Hardmeier, F. Hofer, A. Schwaninger,"The X-ray object recognition test (X-ray ORT) - a reliable and valid instrument for measuring visual abilities needed in X-ray screening," IEEE CCST 2005, pp. 189-192.

[5] Abidi, B., Y. Zheng, A. Gribok and M. Abidi, Screener Evaluation of Pseudo-Colored Single Energy X-ray Luggage Images. Proceedings of IEEE conference on Computer Vision and Pattern Recognition Workshop, San Diego CA June 2005.

[6] B. Abidi, J. Liang, M. Mitckes, and M. Abidi. Improving the detection of lowdensity weapons in xray luggage scans using image enhancement and novel scene-decluttering techniques. Jrnl of Elec. Imaging, 13(3):523-538, 2004.

[7] A. C. Kak and M. Slaney. Principles of computerized tomographic imaging. Society for Industrial and Applied Mathematics, PA, USA, 2001.

[8] S. Singh and M. Singh, "Explosives detection systems(EDS) for aviation security," Signal Processing, vol. 83 , no. 1 , pp. 31-55, 2003.

[9] R. Gesick, C. Saritac, and C. Hung, "Automatic Image Analysis process for the detection of concealed weapon" in Proceedings of the $5^{\text {th }}$ Annual Workshop on Cyber Security and Information Intelligent Research,2009.

[10] V. Muthukkumarasamy, M. Blumenstein, J. Jo, and S.Green, "Intelligent illicit object detection system for enhanced aviation security",in International Conference on Simulated Evolution and Learning,2004.

[11] X.Shi, "Improving object classification in X-ray luggage inspection,Ph.D Thesis,Department of Computer and Electrical Engineering,Virginia Tech.and State University,2000.

[12] T. Feather, I. Guan, A. Lee-Kwen, and R.B. Paranjape, "Caxss: an intelligent threat detection system," SPIE, Applications of Signal and image Processing in Explosives Detection Systems, vol. 1824,pp.152-161, 1993.

[13] A.S.Belongie,J.Malik and J.Puzicha, "Shape matching and object recognition using shape contexts",IEEE Trans.PAMI,vol.24,pp509-522,April 2002.

[14] A.Khotanzad and Y.H.Hong, "Invariant Image Recognition by Zernike Moments",IEEE Transactions on Pattern Anal\& Machine Intelligence,vol.PAMI-12,No.5.,pp.489-497,1990.

[15] Keller, J.M., Gray, R., Givens, J.A.J.R. A Fuzzy k-nearest Neighbor Algorithm. IEEE Trans. Systems Man Cybernet. 15(4), 580-585, 1985.

\section{Authors}

S.Mohamed Mansoor Roomi received his B.E degree in Electronics and communication. Engineering from Madurai Kamarajar University, in 1990 and the M. E (Power Systems) \& ME (Communication Systems) from Thiagarajar College of Engineering, Madurai in 1992\&1997 and phd in 2009 from Madurai Kamarajar University.His primary Research Interests include Image Enhancement and Analysis.

R.Rajashankari received her B.E degree in is in Electronics and Communication. Engineering from Sethu Institute of Technology, Virudhunagar.She is currently doing her M.E degree in Communication systems at Thiagarajar College of Engineering,Madurai,India Her research interests.
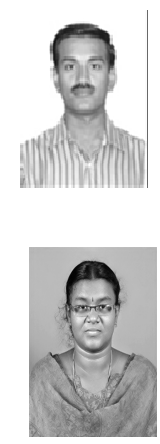